\title{
A COMPUTER SIMULATION MODEL FOR THE BLOW-BLOW FORMING PROCESS OF GLASS CONTAINERS
}

\author{
C. G. Giannopapa \\ Dept. of Mathematics and Computer Science \\ Eindhoven University of Technology \\ PO Box 513, 5600 MB Eindhoven \\ The Netherlands \\ Email: c.g.giannopapa@tue.nl
}

\author{
J. A. W. M. Groot \\ Dept. of Mathematics and Computer Science \\ Eindhoven University of Technology \\ PO Box 513, $5600 \mathrm{MB}$ Eindhoven \\ The Netherlands \\ Email: c.g.giannopapa@tue.nl
}

\begin{abstract}
In glass container manufacturing (e.g. production of glass bottles and jars) an important process step is the blowing of the final product. This process is fast and is characterized by large deformations and the interaction of a hot glass fluid that gets into contact with a colder metal, the mould. The objective of this paper is to extend and further develop our finite element model [1] to be used for industrial purposes. To achieve this both steps of the forming of glass containers, namely blowblow needs to be simulated and tested against real industrial problems. The model should be able to correctly represent the flow of glass, the energy exchange during the process and provide the final thickness of the final product. For tracking the geometry of the deforming inner and outer interface of glass, the level set technique is applied on a fixed mesh. At each time step the coupled problem of flow and energy exchange is solved by the model. Here the flow problem is only solved for the domain enclosed by the mould, whereas in the energy calculations, the mould domain is also taken into account. A non uniform temperature distribution is considered for the blowing of the preform. For all the calculations the material parameters (like viscosity) are based on the glass position, i.e. the position of the level sets. The velocity distribution, as found from this solution procedure, is then used to update the two level sets by means of solving a convection equation. A fast marching re-initialization algorithm is applied after each time step in order to let the level sets re-attain the property of being a signed distance function. The model is validated by several examples focusing on both the overall behavior (such as conservation of mass and energy) and the local behavior of the flow (such as glass-mould contact) and temperature distributions.
\end{abstract}

\section{NOMENCLATURE}

$\begin{array}{lll}A & - & \text { Lakatos coefficients } \\ B & K & \text { Lakatos coefficients } \\ c_{p} & \mathrm{~J} / \mathrm{kgK} & \text { specific heat of glass } \\ f & \mathrm{~m} / \mathrm{s}^{2} & \text { body force (gravitation) } \\ k_{c} & \mathrm{~W} / \mathrm{mK} & \text { conductivity of glass } \\ p & \mathrm{~Pa} & \text { pressure } \\ q & \mathrm{~W} / \mathrm{m}^{2} & \text { heat flux }\end{array}$

$\begin{array}{lll}t & \mathrm{sec} & \text { time } \\ T & \mathrm{~K} & \text { glass temperature } \\ T_{L} & \mathrm{~K} & \text { Lakatos coefficients } \\ U & \mathrm{~m} / \mathrm{sec} & \text { velocity } \\ \eta & \mathrm{m}^{2} / \mathrm{s} & \text { dynamic viscosity } \\ \rho & \mathrm{kg} / \mathrm{m}^{3} & \text { density } \\ \sigma & \mathrm{Pa} & \text { Cauchy stress tensor }\end{array}$

\section{INTRODUCTION}

For centuries glass forming of containers such as bottles, jars and art articles, was performed by hand and was based on empirical knowledge and hands on experience. In the $19^{\text {th }}$ century glass blowing was made by compressed air and automation. There has been a significant progress in understanding and optimizing the glass manufacturing process [2,3]. Computer model simulations become more and more important in this process as they give an insight of the phenomena happening fast and at extremely high temperature conditions.

In [1] the development of a computer model to be used for glass blowing was described and demonstrated on dummy problems. This paper concentrates on modeling the full blowblowing forming process of glass containers with realistic data provided by glass manufacturers. In particular it is focused on the accurate representation of the glass-air interface during blowing and the thickness of the final product.

For glass containers manufacturing, the forming process is typically comprised of two stages: either press-blow or blowblow. A schematic of the blow-blow process can bee seen in Figure 1. When the molten glass leaves the furnace where temperature goes up to $1400{ }^{\circ} \mathrm{C}$ it goes through the feeder and it is cut into uniform gobs of glass by a shearing and distribution system. Each gob is sent to an individual section forming machine where the temperature drops below $1150{ }^{\circ} \mathrm{C}$, which forces the gobs to take the moulds shape. The forming machine consists of two moulds. In the first blow mould the gob drops from above (Figure 1a) and pressurized air blows the gob to the base of the mould to form the neck of the bottle (Figure 1b). Compressed air is then blown from the bottom of the mould forcing the inner part of the gob to rise and take the shape of the mould (Figure 1c) forming a thick walled preform or parison. This parison is removed by a robotic arm from the first 
mould, is turned upside down and is transferred to a second mould (Figure 1f). The preform sags due to gravity sufficiently far without touching the bottom of the mould. Finally pressurized air is used to inflate the preform to the final container shape (Figure 1e). The container is then removed from the mould and transferred to an annealing oven where it is reheated to remove the stress produced during forming and is then cooled under controlled conditions. (a)

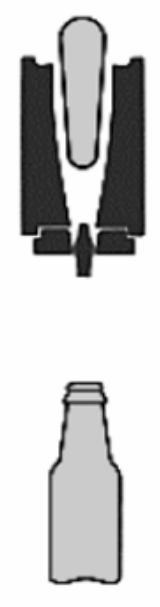

(f) (b)
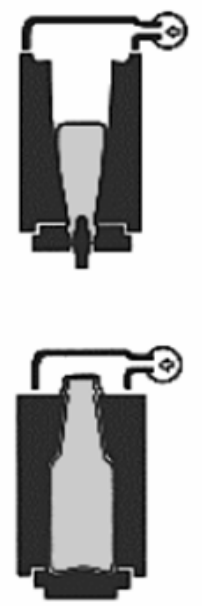

(e) (c)

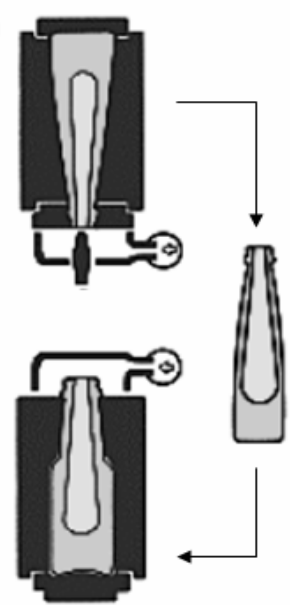

(d)
Figure 1: Blow-blow process

In spite of the fact that the glass manufacturing process has been used for many years, glass manufacturers still experience difficulties in optimizing and controlling this process. Computer simulations would be very helpful to gain a better insight and assist in designing optimal bottle shapes. Simulations offer a good alternative to time consuming and expensive trial and error procedures common to factories. Representative numerical simulations could help minimize unwanted variations in wall thickness of containers and reduce their weight while maintaining the strength. They can also help optimize cooling conditions and increase the production speed. All this could result in decreasing manufacturing cost.

In correctly modeling the blow stage of the forming process one has to accurately capture the glass-air interface. There is a number of numerical techniques that advocate for solving moving interface problems. They can be classified into two main categories: interface-tracking techniques (ITT) and interface-capturing techniques (ICT).

\section{Interface-tracking techniques}

The interface tracking techniques (ITT) are based on the Deforming-Spatial-Domain/Stabilized Space-Time formulation, where the mesh is updated as the flow evolves in order to track the interface $[4,8]$. The major challenge of this method is the mesh update. They focus on how the mesh should be updated to accommodate for the changes in the spatial domain which depends on several factors such as the complexity of the interface and the overall geometry, how unsteady the interface is and how the initial mesh was generated. Mesh updating can become a very computationally expensive matter especially when the number of elements becomes very large or when the frequency of re-meshing has to be high. In the mesh moving strategies, the only rule in the mesh motion one needs to follow is that at the interface the normal velocity of the mesh has to match the normal velocity of the fluid. The strongest challenge in mesh updating techniques is to minimize the frequency of remeshing.

\section{Interface-capturing techniques}

The interface-capturing techniques (ICT) functions on fixed meshes. These methods are based on formulations, of both the flow equations and the advection equation governing the time-evolution of an interface function. The interface function is the one that marks the location of the interface. In these methods the major concern is the accurate representation of the interface. This involves the accurate representation of the interface geometry as well as the representation of the flow near the interface. The interface is captured within the resolution of the mesh used. The interface capturing techniques use a massless particle or an indicative function to mark the continuum on either side of the interface. It is based on an Eulerian approach where the mesh once generated is maintained and gives the flexibility to apply it in complicated topologies; thus, it is very attractive for this project. The main interface capturing techniques are the Volume-of-Fluid method [9] and Level Set Method [10,11].

As this project is driven by industry, computational time is very essential. The interface tracking techniques due to the computational overhead related with the re-meshing are not as attractive for this application as the interface capturing techniques. The Level Set Method has been chosen for the interface tracking in this project.

The objective of this paper is to create a robust computational model to be used for industrial purposes which can accurately capture the blow-blow forming of glass containers. The finite element model should be able to correctly represent the free surface flow of glass with high temperature dependent viscosity and the energy exchange during the process. The model should be able to take as input information the gob volume or the glass preform shape, a temperature distribution of the gob or preform and the mould and a prescribed inlet air pressure. It should output the containers preform or final shape, the products final wall thickness as well as the stress and thermal deformations the pre-form and the mould undergo during the process.

\section{MATHEMATICAL FORMULATION}

This section is concerned with presenting the mathematical model used to describe the blowing stage of the production process. This involves solving the motion of the glass, the heat exchange in the glass and the position of the glass-air interface.

\section{Flow problem}

The motion of the hot glass can be described by the Navier-Stokes equations for fluids.

Momentum equation:

$\rho\left(\frac{\partial \mathbf{U}}{\partial t}+\nabla \cdot(\mathbf{U U})\right)=\nabla \cdot \boldsymbol{\sigma}+\rho \mathbf{f} \cdot$

Continuity equation or mass conservation:

$\nabla \cdot \mathbf{U}=0$. 
For Newtonian fluids the constitutive equation for the stress tensor, $\boldsymbol{\sigma}$ is given by:

$\boldsymbol{\sigma}=2 \eta \operatorname{dev}(\dot{\boldsymbol{\varepsilon}})-p \mathbf{I}=\eta\left(\nabla \mathbf{U}+\nabla \mathbf{U}^{T}\right)-p \mathbf{I}$

where $\varepsilon$ is the strain tensor, $\dot{\varepsilon}$ is the strain rate tensor and $\operatorname{dev}($.$) is the deviatoric part of the tensor. The dynamic viscosity$ depends on the position

$\eta=\eta(x)$.

Substituting (3) into (1) the momentum equation can be rewritten as:

$\rho \frac{\partial \mathbf{U}}{\partial t}=\nabla \cdot(\eta \nabla \mathbf{U})-\rho \nabla \cdot(\mathbf{U U})-\nabla p+\rho \mathbf{f}$

In order to further analyze the problem quantitatively equation (5) is made dimensionless. Let us consider a typical: velocity $\mathbf{U}_{0}$, length scale $L_{0}$, viscosity $\eta_{0}$, density $\rho_{0}$, and pressure $\mathrm{p}_{0}$. The characteristic pressure for the glass domain can be derived from:

$p_{0}=\eta_{0} U_{0} / L_{0}$,

and for the air domain by:

$p_{0}=\rho_{0} \mathbf{U}_{0}{ }^{2}$.

Defining dimensionless time, length, velocity and pressure:

$t^{*}=\frac{\mathbf{U}_{0} t}{L_{0}}, x^{*}=\frac{x}{L_{0}}, \mathbf{U}^{*}=\frac{\mathbf{U}}{\mathbf{U}_{0}}, p^{*}=\frac{p}{p_{0}}$.

Define dimensionless gravitational body forces.

$\mathbf{f}^{*}=\frac{\mathbf{f}}{\|\mathbf{f}\|}$.

The dimensionless form of the momentum (Equation 5) and the continuity equation (Equation 2) are:

$\rho^{*} \frac{\partial \mathbf{U}^{*}}{\partial t^{*}}=\frac{1}{\operatorname{Re}} \nabla \cdot\left(\eta^{*} \nabla \mathbf{U}^{*}\right)-\rho^{*} \nabla \cdot\left(\mathbf{U}^{*} \mathbf{U}^{*}\right)-\frac{p_{0}}{\rho_{0} \mathbf{U}_{0}^{2}} \nabla p^{*}+\frac{1}{F r_{2}} \rho^{*} \mathbf{f}^{*}$

$\nabla \cdot \mathbf{U}^{*}=0$.

Here the relation between the internal and viscous forces is described by the Reynolds number $R e=\rho_{0} U_{0} / \eta_{0}$ and the ratio of the internal force to the gravitational force is give by the Froude number $F r_{2}=\mathbf{U}_{0}^{2} /\|\mathbf{f}\| L_{0}$.

\section{Heat exchange}

The heat flow can be described by the energy equation and for an incompressible fluid in an Eulerian description it reads:

$\rho c_{p}\left(\frac{\partial T}{\partial t}+\mathbf{U} \cdot \nabla T\right)=-\nabla \cdot \mathbf{q}$.

Here $c_{p}$ is the specific heat of the glass, $q$ is the heat flux and $\phi$ is the source term from the internal heat generation by viscous and volume forces. Neglecting the heat transfer due to radiation and assuming that the conduction obeys the Fourier law which relates the heat flux vector to the temperature $\mathrm{T}$ by thermal conductivity $\mathrm{k}$ as

$\mathbf{q}=-k \nabla T$

Thus, the energy equation can be written as:

$\rho c_{p}\left(\frac{\partial T}{\partial t}+\mathbf{U} \cdot \nabla T\right)=k \nabla^{2} T$.
Define dimensionless viscosity, density, conductivity and specific heat:

$\eta^{*}=\frac{\eta}{\eta_{0}}, \rho^{*}=\frac{\rho}{\rho_{0}}, \kappa^{*}=\frac{\kappa}{\kappa_{0}}, c^{*}=\frac{c_{p}}{c_{0}}$

Define dimensionless temperature:

$T^{*}=\frac{T-T_{0}}{\Delta T}$,

where $\mathrm{T}_{0}$ is the temperature at the mould and $\Delta \mathrm{T}=\mathrm{T}_{\mathrm{g}}-\mathrm{T}_{0}$ is the temperature jump between the glass and the mould.

The dimensionless form of the energy equation (14) reads:

$P e \rho^{*} c_{p}^{*}\left(\frac{\partial T^{*}}{\partial t^{*}}+\mathbf{U}^{*} \cdot \nabla \mathbf{U}^{*}\right)=k^{*} \nabla^{2} T^{*}$,

where the forced convection of a system is related to its heat conduction by the Péclet number $P e=\rho_{0} c_{p} L_{0} U_{0} / k_{0}$.

It should be mentioned that the viscosity $\eta$ typically represents the fluids' resistance to the flow. In the glass the viscosity strongly depends on the temperature. As the temperature rises the viscosity decreases. Its values can be obtained by the so-called Vogel-Fulcher-Tammann (VFT) relation [2]:

$\eta(T)=10^{-A+B /\left(T-T_{L}\right)}$

where $\mathrm{A}, \mathrm{B}, \mathrm{T}_{\mathrm{L}}$ are the Lakatos coefficients and are empirically determined depending on the type of the glass.

\section{Glass-air interface position - Level set method-}

The problems this paper intends to model involves moving interfaces. The level set method has been chosen to capture the moving interface between the two continua, glass and air $[10,11]$. This method allows to model flows with moving interfaces using a fixed spatial domain. Two continua A and B with different densities and viscosities are considered.

The basic idea is to embed a moving interface as the zero level set of the interface function $\phi$, which serves as a marker identifying the interface between the two continua. The evolution of the zero level set, thus the motion of the interface, is governed by the convention equation:

$\frac{\partial \phi}{\partial t}+\mathbf{U} \cdot \nabla \phi=0$

So, at any time t the corresponding interface $\Gamma(\mathrm{t})$ is given implicitly by $\phi(x, t)=0$. In this project, for the position of the glass-air interface two level sets are used, one describing the inner interface of the perform and the other the outer one.

\section{DISCRETISATION METHOD}

The finite element (FE) method has been used for the discretization of the Stokes problem, the level set functions and the energy equation. The finite-element formulation has been implemented in a SEPRAN finite element package. The mini element is used for the computations [14].

The resulting equations for the Stokes problem are solved using the BI-CGSTAB method [20]. This iterative method terminates after a finite number of iteration steps. As a stepping criterion the absolute value of the residual less than $10^{-8}$ is used. 
As an initial guess for the iteration process the solution obtained in the previous time step is used.

The stabilized formulation of the energy equation is obtained using the streamline-upwind Petrov-Galerkin (SUPG) method $[15,21]$. The BI-CGSTAB method [20] is used to solve the equations up to an accuracy of $10^{-6}$. As before, the values obtained from the previous time step are used as an initial guess. For the level set solution the accuracy used is $10^{-8}$. The Euler Implicit scheme was used for the time integration.

The Level Set Method is used for capturing the two-fluid flow interface. It is adequate to compute geometric properties of highly complicated boundaries without necessarily explicitly tracking the interface. So the moving interface can undergo topological changes i.e. develop corners quite naturally.

One of the difficulties encountered in this method is to set the level set function keeping the desired shape. When the velocity in the domain is not constant the function itself can deform such that it introduces additional numerical problems. There are two solutions to this problem: (a) to adapt the velocity field, this procedure of extension velocities, is mainly used for problems where the velocity is only known at the interface; (b) to re-initialize the level set function, in this case the velocity field is unchanged. In the case concerned it is chosen to keep the velocity field unchanged and re-initialize the level set function.

The level set function $\phi$ is initially a Euclidean distance function to the corresponding interface $\Gamma(\mathrm{t})$ where

$\|\nabla \phi(\mathbf{x}, 0)\|=1$.

While the interface is convected by the interface velocity according to Equation (19), the gradient of the function $\phi$ is not equal to 1 and $\phi$ may become irregular after some time. The initially well shaped level set function can develop steep gradients on one side and can become almost constant on the other side of the domain. Thus, it is desirable to compute a function $\mathrm{d}(\mathbf{x})$, that has the following property while the zero level of the distance function coincides with the interface

$\|\nabla d(\mathbf{x})\|_{2} \leq 1$.

The re-initialization used in this paper is based on fast marching methods and can be used for computations in both structured and unstructured meshes.

The fast marching methods [17,18] solve the following boundary value equations in the computational domain:

$\|\nabla d(\mathbf{x})\|_{2}=1$,

$d(x)=0 \quad$ on $\Gamma$.

The fast marching level set method is based on the idea that the surface front is propagating outward with a velocity in an upwinding fashion. The interface is carried along the computational domain by considering a narrow band around it and marching along the grid points. During this propagation some of the values of the grid points that start getting further away from the narrow band around the interface become irrelevant and are 'frozen' and new ones that are coming closer are included.

Figure 2 represents the algorithmic representation of the method where a two dimensional grid is considered with the dashed line representing the location of the interface (Figure 2a). The distance between two grid points is $\Delta x$. The algorithm is initialized [19] by computing the $d$ at grid points close to the interface $\Gamma$. These immediate points around the interface are tagged as "proximate" and in Figure $2 \mathrm{~b}$ are color marked as grey. All neighbor points to the "proximate" points have known values and are tagged as "known" and color marked in Figure $2 \mathrm{c}$ as white. Points located further than the "known" points are marked as "far" and are not of interest. The ones that are in the upfront of the interface will become of interest as the interface propagates. The points that are candidates to be added to the set of "known" points of to the set of "far" points are the "trial" points. These points have a trial value $d$ assigned to them that might not yet be the correct one.

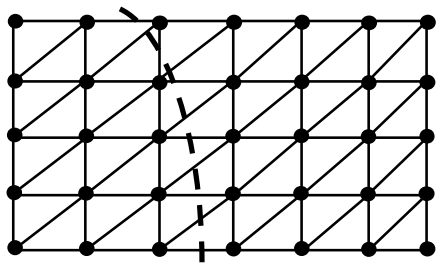

(a)

(c)

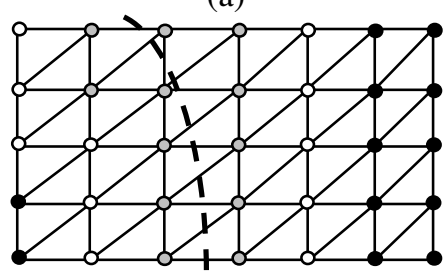

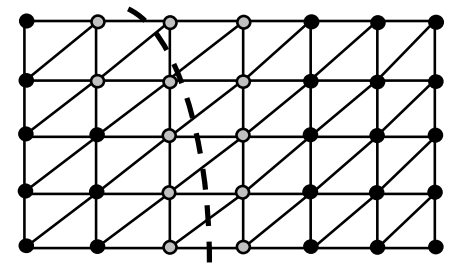

(b)

(d)

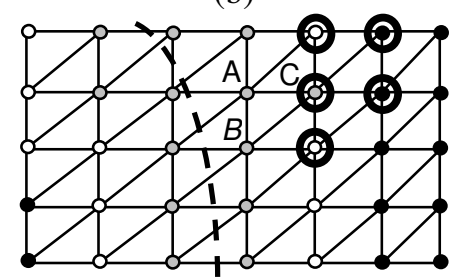

Figure 2: Fast marching methods.

Point $\mathrm{C}$ in Figure 2d with two known neighbors $\mathrm{A}$ and $\mathrm{B}$ is considered. The trial value at this point considering that $d_{C} \geq d_{A}$, $\mathrm{d}_{\mathrm{B}}$ is the solution of the quadratic equation:

$\left(d_{C}-d_{A}\right)^{2}+\left(d_{C}-d_{B}\right)^{2}=\Delta x^{2}$.

After having assigned values $d$ to all the "trial" points, the points with the smallest value are the ones with the correct value. If this is the case for the point $\mathrm{C}$ it can be removed from the "trial" set of points and be added to the "known" one.

Thus, the algorithm can further proceed with the neighbors of point $\mathrm{C}$ denoted in Figure $2 \mathrm{~d}$ by the big circles. In this case the "far" points are transferred to the "trial" point set and trial values of $d$ are computed.

\section{COMPUTER SIMULATION MODEL}

The blow-blow process was modeled. The simulations considered are tested on commercial bottle containers. Simulations were performed both for the first blow as well as the second blow. The preform's outer shape in the second blow has been used as the mould shape for the first blow step. The information regarding the dimensions and the manufacturing conditions were supplied by manufacturers.

In the first blow mould we do not consider the neck formation and the initial compression of the gob by pressurized air. Our simulation starts after the gob is settled. The glass movement is captured by the level sets.

The typical values used in the simulations are:

$\rho_{0 \text { (glass) }}=1.0 \mathrm{~kg} / \mathrm{m}^{3}, \quad \rho_{0 \text { (glass) }}=2.53 \cdot 10^{-3} \mathrm{~kg} / \mathrm{m}^{3}, \quad p_{0}=1.38 \cdot 10^{5} \mathrm{~Pa}$ $\mathrm{c}_{\mathrm{p} \text { (glass) }}=1.4 \cdot 10^{3} \quad \mathrm{~J} /(\mathrm{kg} \cdot \mathrm{K}), \quad \mathrm{c}_{\mathrm{p}(\text { air })}=10^{3} \quad \mathrm{~J} /(\mathrm{kg} \cdot \mathrm{K}), \quad \mathrm{k}_{\text {glass }}=3 \cdot 10^{-}$ ${ }^{2} \mathrm{~W} /(\mathrm{m} \cdot \mathrm{K}), \mathrm{k}_{\mathrm{air}}=3 \cdot 10^{-2} \mathrm{~W} /(\mathrm{m} \cdot \mathrm{K})$. 
The momentum equation (Equation 10) can be simplified based on the following arguments. The Reynolds number in the glass domain is $2.53 \cdot 10^{-5}$. For the simulations the air is replaced by a fictitious fluid with viscosity $2.1 \mathrm{~Pa} \cdot \mathrm{s}$ and density $1 \mathrm{~kg} \cdot \mathrm{m}^{-3}$. For the fictitious fluid the Reynolds number is $4.76 \cdot 10^{-5}$. As $\mathrm{Re}<<1$ the problem is dominated by viscous and gravitational forces and the inertia terms may be neglected. Thus, the flow problem can be simplified by solving the Stokes equation.

Two level set functions were used to capture the moving interface; one for the outer interface and one for the inner interface. Simulations were performed using both structured and unstructured meshes. Graphs of the finite element mesh can be found in Figure 3.

An inflow pressure is prescribed at the boundary and a free-stress condition applies to the air at the walls of the mould. In the quarter circle the glass can freely move outside the computational domain whereas in the case of the half bottle noslip condition applies at the mould walls.

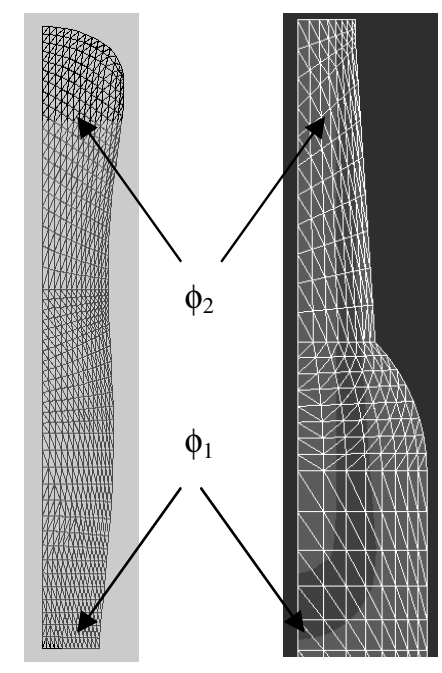

(a)

(b) of air is depicted with blue. The temperature profiles can be seen in Figure 5.
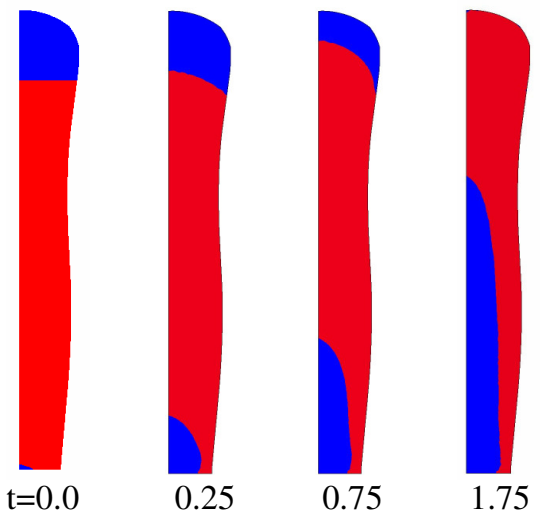

\section{Figure 4: The glass area propagation of the first blow step of the preform. Air is denoted with blue and glass with red.}

In the second blow step the preform is first let inside the mould to stretch due to gravity long enough but without touching the mould. The stretch time used for the simulations was $0.3 \mathrm{~s}$. After this time the pressurized air forces the preform to obtain the shape of the mould. The glass propagation during stretch and blow are shown in Figure 6. In Figure 7 the temperature profiles are shown. The thickness of the final product can be seen in Figure 8.

\section{Figure 3: Finite element mesh and interface representation: (a) preform (b) bottle.}

It is common practice in glass manufacturing that there is a temperature variation in the glass preform, which results in wall thickness variations. Thus a non-uniform distribution was considered for the second blow of the preform.

The temperature distribution is give by:

$$
T(x, y)=T_{0}\left(1-\lambda_{y} e^{-k_{y} y}\right)+\sum_{k=1}^{4}(-1)^{k}\left(T_{k, x} x^{k}+T_{k, y} y^{k}\right)
$$

where $\mathrm{T}_{0}=3.2 \cdot 10^{3}, \mathrm{~T}_{1, \mathrm{x}}=6.9 \cdot 10^{4}, \mathrm{~T}_{2, \mathrm{x}}=1.7 \cdot 10^{7}, \mathrm{~T}_{3, \mathrm{x}}=1.3 \cdot 10^{9}$, $\mathrm{T}_{4, \mathrm{x}}=3.2 \cdot 10^{10}, \quad \mathrm{~T}_{1, \mathrm{y}}=4.4 \cdot 10^{4}, \quad \mathrm{~T}_{2, \mathrm{y}}=4.3 \cdot 10^{5}, \quad \mathrm{~T}_{3, \mathrm{y}}=7 \cdot 10^{5}, \quad \mathrm{~T}_{4, \mathrm{y}}=0$, $\lambda_{\mathrm{y}}=3.4 \cdot 10^{5}, \kappa_{\mathrm{y}}=200$.

\section{RESULTS}

For the simulations of the first blowing step the initial temperature of air and the mould were $500{ }^{0} \mathrm{C}$. The initial temperature of the gob was considered to be uniform. The propagation of the glass during the forming process can be seen in Figure 4. The area of glass is depicted with red and the area

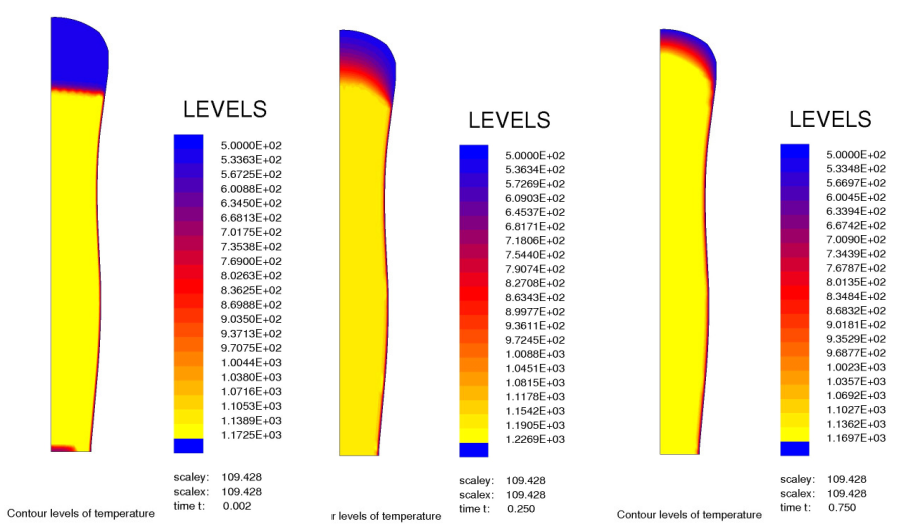




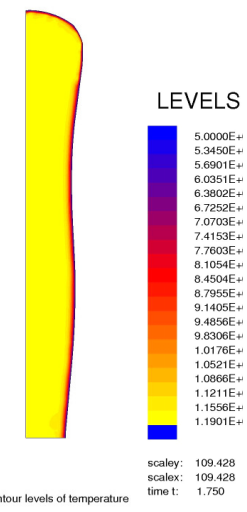

Figure 5: Temperature profiles of the first blow step of the preform at different times.

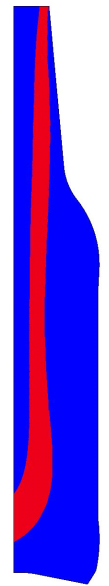

0.3

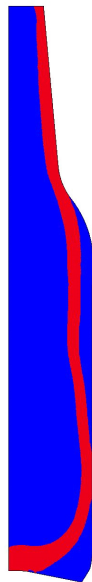

0.725

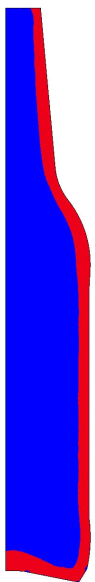

1.025
Figure 6: The glass area propagation of the second blow step of the preform with stretch time $0.3 \mathrm{~s}$. Air is denoted with blue and glass with red.

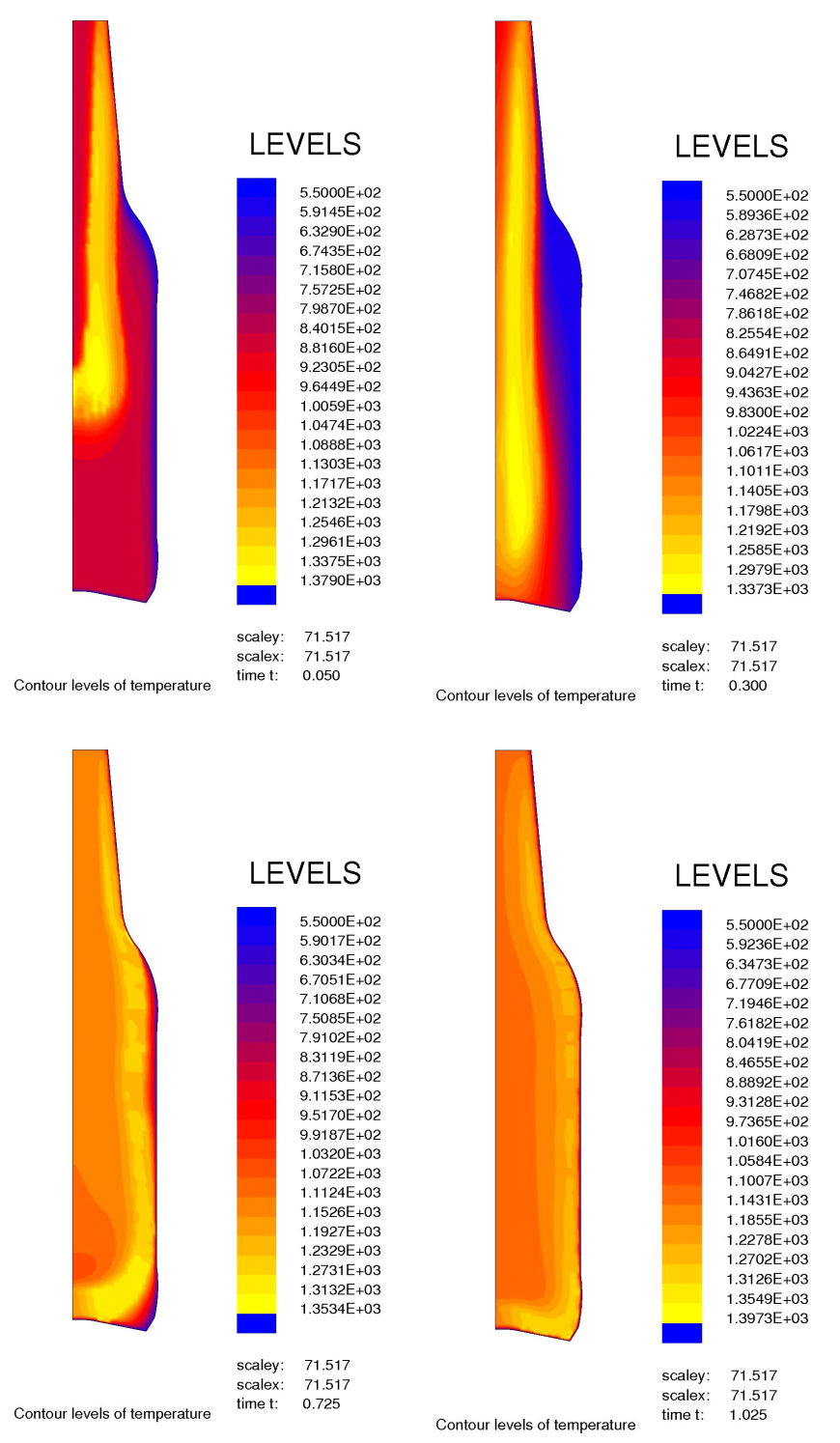

Figure 7: Temperature profiles of the second blow step of the preform with stretch time $0.3 \mathrm{~s}$ at different times. 


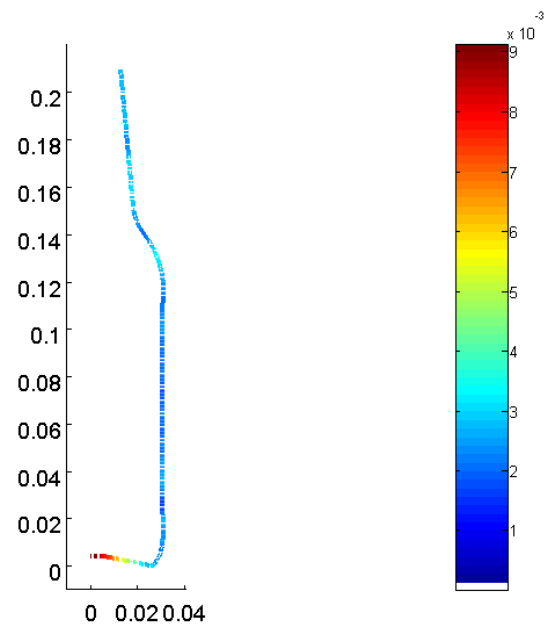

Figure 8: Final product thickness distribution [m].

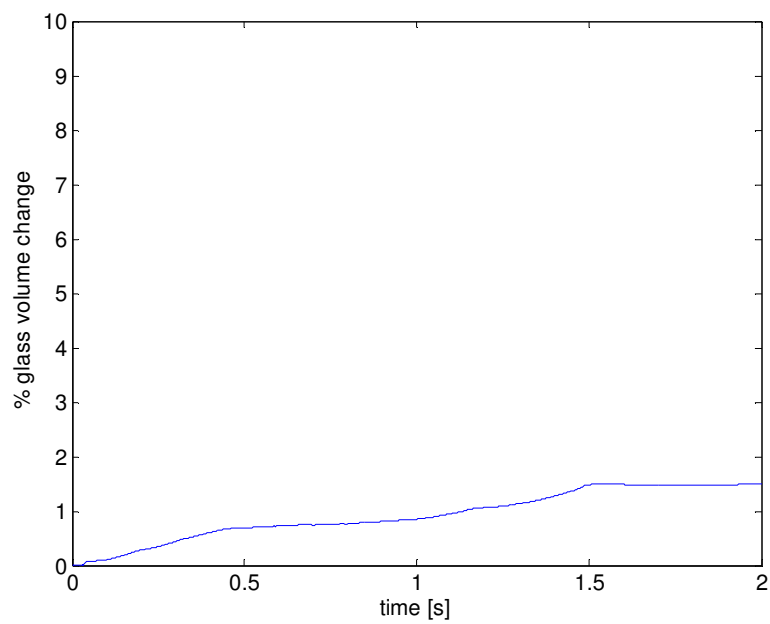

Figure 9: Volume conservation of the first blow and second blow step of glass containers using Euler Implicit scheme with time step 1e-4.

In order to assess the accuracy of the simulation model presented in this paper, the glass volume conservation is used. In Figure 9 the percentage volume change in time is shown. The volume change has a maximum of $1.5 \%$. From previous investigation [3] we have seen that the volume conservation can be further improved using smaller time steps, higher mesh quality and a second order accurate discretisation schemes such as Crank Nicholson.

\section{CONCLUSIONS}

In this paper we presented the implementation of the forming process of glass containers tested in realistic manufacturing conditions. Both the first and the second blow step of the forming process were modeled. A realistic nonuniform temperature distribution of the preform in the second step was modeled and the final shape of the end product was presented.

The fluid flow is described by the Stokes equations and the temperature by the energy equations. The glass position is modeled using two level set function representing the inner and outer glass-air interface.

The method was implemented in a finite elements library and can work for both structured and unstructured grids giving flexibility to manufacturers to use it for any form and shape the end product might have.

The method gave a volume conservation of between $1.5 \%$ which can further be improved using higher order discretisation schemes, which gives confidence that this model can be used for industrial practice. The next step of development is to compare it with experimental data provided by industry.

\section{ACKNOWLEDGMENTS}

The authors would like to thank Marie Currie Research Training Networks Fellowships, European Commission for funding this project.

\section{REFERENCES}

[1] Giannopapa C. G., 2006, "Development of a computer simulation model for blowing glass containers", ASME PVP 2006, Vancuver, BC, Canada, PVP2006-ICPVT11-93262.

[2] Tooley, F. V., 1984, "The handbook of glass manufacture", Vol II, Aslee Publishing Co.. New York.

[3] Loch, H. , Krause, D. , 2002, "Mathematical simulation in glass technology", Springer-Verlag Berlin and Heidelberg GmbH \& Co. K.

[4] Hirt, C.W., Cook, J.L., Butler, T.D, 1970, “A Lagrangian method for calculating the dynamics of an incompressible fluid with free surface", J. Computational Physics ,5, p.103-124.

[5] Radovitzky, R., Ortiz, M., 1998, "Lagrangian finite element analysis of newtonian fluid flows", Int. J. Numerical Methods in Engineering, 43, p.607-619.

[6] Hirt, C.W., Amsden, A.A., Cook, J.L., 1974, "An arbitrary lagrangian - eulerian computing method for all flow speeds", $J$. Computational Physics, 14, p.227-253.

[7] Masud, A., Hughes, T.J.R., 1997, "A space - time Galerkin/least - squares finite element formulation of the navierstokes equations for moving domain problems", Computational Methods in Applied Mechanics and Engineering, 146(1-2), p.91-126.

[8] Souli, M., Zolesio, J.P., 2001, "Arbitrary LagrangianEulerian and free surface methods in fluid mechanics", Computational Methods in Applied Mechanics and Engineering, 191, p.451-466.

[9] Hirth, C.W., Nichols, B.D., 1981, "Volume of fluid (VOF) method for the dynamics of free boundaries", J. Comp. Phys., 39, 201-225. 
[10] Sethian, J.A, 1999, "Level Set Methods and Fast Marching Methods", Cambridge university press, USA.

[11] Sussman, M., Smereka, P., Osher, S.J., 1994, "A level set method for computing solutions to incompressible two-phase flow", J. Comp. Phys, 114, 146-159.

[12] Cuvelier, C., Segal, A., van Steenhoven, A., 1986, "Finite Element Methods and Navier Stokes Equations, Mathamatics and its applications", D. Reidel Publishing Co., The Netherlands.

[13] Gunzberger, M., 1989, "Finite element methods for viscous incompressible flows", Computer Science and Scientific Computing, Academic Press.

[14] Bathe, K. J., 1997, "Finite element procedures", Prentice Hall, Englewood Cliffs, New Jersey.

[15] Johnson, C., Nävert, U., Pitkäranta, J., 1984, "Finite element methods for linear hyperbolic problems", Computer methods in applied mechanics and engineering, 45, 285-312.

[16] L. A. Hageman and D. Young, 1981, "Applied Iterative Methods", 1st ed. New York: Academic.

[17] Sethian, J.A., 1996, "A marching level set method for monotonically advancing fronts", Proc. Nat. Acad. Sci., 93, 1591-1595.

[18] Sethian, J.A., 1999, "Fast marching methods", SIAM review, 41(2), 199-235.

[19] Chopp, D.L, 2001, "Some improvements of the fast marching method", SIAM Journal of Scientific Computing, 23(I), 230-244.

[20] van der Vorst. H.A., 1992, "BI-CGSTAB: A fast and smoothly converging variant of BI-CG for the solution of nonsymmetric linear systems", SIAM J. Sci. Stat. Comput, 13(2), 631-644.

[21] Brooks, A.N., Hughes, T.J.R., 1982, "Stream-line upwind/Petrov-Galerking formulation for convection dominated flows with particular emphasis on the imcompreible Navier-Stokes equations", Comput. Methods Appl. Mech. Eng., 32, 199-259.

[22] Shakib, F., 1989, "Finite element analysis of the compressible Euler and Navier-Stokes equation", PhD thesis, Stanford University. 